\title{
Medium Spatial Frequencies, a Strong Predictor of Salience
}

\author{
Fabrice Urban • Brice Follet • Christel Chamaret • \\ Olivier Le Meur · Thierry Baccino
}

Received: 30 April 2010/Accepted: 8 November 2010/Published online: 23 November 2010

(C) Springer Science+Business Media, LLC 2010

\begin{abstract}
The extent to which so-called low-level features are relevant to predict gaze allocation has been widely studied recently. However, the conclusions are contradictory. Edges and luminance contrasts seem to be always involved, but literature is conflicting about contribution of the different spatial scales. It appears that experiments using man-made scenes lead to the conclusion that fixation location can be efficiently discriminated using high-frequency information, whereas mid- or low frequencies are more discriminative for natural scenes. This paper focuses on the importance of spatial scale to predict visual attention. We propose a fast attentional model and study which frequency band predicts the best fixation locations during free-viewing task. An eye-tracking experiment has been conducted using different scene categories defined by their Fourier spectrums (Coast, OpenCountry, Mountain, and Street). We found that medium
\end{abstract}

\author{
F. Urban $(\bowtie) \cdot$ B. Follet · C. Chamaret \\ Technicolor Research and Innovation, Video Processing \\ and Perception Lab, 1 av. de belle Fontaine, CS 17616, \\ 35576 Cesson Sévigné CEDEX, France \\ e-mail: fabrice.urban@technicolor.com \\ B. Follet \\ e-mail: Brice.Follet@ technicolor.com \\ C. Chamaret \\ e-mail: Christel.Chamaret@technicolor.com \\ O. Le Meur \\ Université de Rennes 1, Campus Univ. de Beaulieu, \\ 35042 RENNES Cedex, France \\ e-mail: olemeur@irisa.fr \\ B. Follet $\cdot$ T. Baccino \\ LUTIN (UMS-CNRS 2809), Cité des sciences et de l'industrie \\ de la Villette, 30 av. Corentin Cariou, 75930 Paris, France \\ e-mail: baccino@lutin-userlab.fr
}

frequencies (0.7-1.3 cycles per degree) globally allowed the best prediction of attention, with variability among categories. Fixation locations were found to be more predictable using medium to high frequencies in man-made street scenes and low to medium frequencies in natural landscape scenes.

Keywords Attention - Saliency map - Bottom up · Scene category $\cdot$ Computational modeling $\cdot$ Eye tracking

\section{Introduction}

Visual attention is one aspect of our visual system used to deal with the large amount of visual data present in our visual environment. Focusing only on visually important areas of our visual field, for a given task or because of their salience, is a very efficient way to decrease the amount of data that the brain has to process. This process of concentrating our attentional resources on restricted areas aims at maximizing information sampled from our visual field. Salience is a prediction of where we look at. Computational saliency models such as the one by Itti et al. [1] are known to provide good saliency maps. Such model provides a saliency map that simulates the computation of primary visual areas by the integration of different parallel feature channels at various spatial scales. In order to understand the role and the respective importance of each feature, different authors have investigated image feature characteristics and their impact on ocular behavior.

Reinagel and Zador [2] outlined that fixated regions have high spatial contrast. Baddeley and Tatler [3] showed that high-frequency edges allow stronger discrimination of fixated over non-fixated regions. Meanwhile, Parkhurst et al. [4] showed that luminance and contrast are more 
predictive than orientation. Tatler et al. [5] found that contrast and edge information were more discriminatory than luminance and chromaticity. They also showed an increased predictability at high frequencies.

Although these studies [3,5] seem to demonstrate a greater visual attraction to high frequencies, other works in the literature favor medium frequencies. By comparing Fourier spectrums of image patches at fixated areas to those at random positions, Bruce et al. [6] found that fixated locations had more horizontal and vertical frequency content then random positions. The more noticeable difference was for medium frequencies. Açik et al. [7] demonstrated that an increase in luminance contrast in natural images favors gaze attraction, whereas the inverse effect appears when decreasing local luminance contrast. However, this effect has not been reproduced for fractal images. More interestingly, after low-pass filtering images, luminance contrast explained fixation locations better in the case of natural scenes and slightly worse in the case of urban scenes. The authors put forward that the attention system might still consider reasonably low frequencies only, in order to cover the full range of saccade length.

In natural images, the amplitude frequency spectrum follows a 1/f law [8]. Low frequencies represent more energy than other frequency levels and are susceptible to be processed more quickly $[9,10]$. Visual scene can be automatically categorized by the brain in $150 \mathrm{~ms}$ before eye movement can occur [9]. Without scene scanning, the scene recognition may involve parafoveal vision which provides only low-frequency information [11]. Other studies show that low spatial frequency information is involved in scene recognition process [12] and facilitates fast recognition of scenes in a coarse-to-fine way $[12,13]$.

The relation between frequency range and visual importance appears to be not well defined and even conflicting in the literature. In addition, conclusions seem to be related to the images used and suggest a variation of attentional behavior according to image semantic context.
In the present paper, we investigate the impact of the different frequency bands on the gaze allocation. We will describe here an experimental framework in order to investigate the importance of spatial scale in the prediction of gaze allocation. Our proposed model is used to compute saliency maps from different early visual features at different scales. An eye-tracking experiment has been conducted to record fixation location on a database of 120 images evenly distributed in 4 semantic categories based on spectral definitions from the work of Torralba et al. [14].

\section{Methods}

Bottom-Up Modeling of the Visual Attention

The computational model of visual attention described in this paper is based on the plausible neural architecture of Koch and Ullman [15]. Numerous models based on this approach have been developed previously [1, 16, 17]. Following this plausible architecture, a simple and computationally efficient bottom-up model has been designed and is used in this paper. Due to the involved experiments, the need for controlling different aspects of the software was strong: the proposed model can output intermediary feature maps for experimental purpose. An additional strong constraint for the developed model is to keep the computational complexity within an acceptable range for possible future real-time implementation (the execution time is currently less than $0.2 \mathrm{~s}$ to process an $800 \times 600$ image). We demonstrate (see Results) that the performances of the proposed model are similar to or even higher than state-of-the-art models in terms of prediction.

The visual attention model uses a hierarchical decomposition of the visual signal. Its synoptic is described in Fig. 1. The YUV 4:2:0 color space is used. It separates achromatic (Y) and chromatic (U: green-magenta and V: orange-cyan) perceptual signals, with chromatic components having half the spatial resolution of achromatic component. The color space has been chosen because it
Fig. 1 Overview of the proposed visual attention model

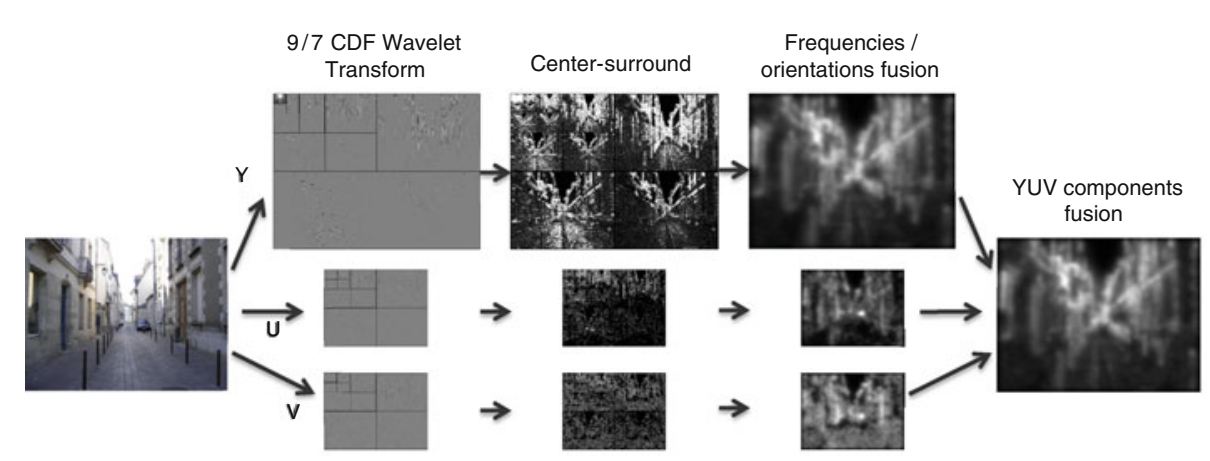


takes the human visual system into consideration and is commonly used in image and video processing.

Psychophysics experiments showed that in TV-viewing condition, the signal can be decomposed in psychovisual channels [18]. Each channel consists in specific frequency and orientation ranges, in achromatic and chromatic components. The achromatic component has a more complex decomposition than chromatic components and also uses higher frequencies. The YUV color space, 4:2:0 sampling, and wavelet decomposition are used here as an approximation of this decomposition. It is also based on the "cortex transform" [19]. As the processing of $\mathrm{Y}, \mathrm{U}$, and V components follows the same scheme, with reduced scale for $\mathrm{U}$ and $\mathrm{V}$, only the processing of $\mathrm{Y}$ channel is described below.

The first step of the model is to extract early visual features from the image. A 9/7 Cohen-Daubechies-Feauveau $(\mathrm{CDF})$ wavelet transform is used to separate frequency bands and orientation ranges. The wavelet transform separates frequencies with a succession of lowpass and high-pass filters followed by critical sub-sampling. The resulting multiscale pyramid is composed of oriented contrast maps with limited frequency range and a low-resolution image. One wavelet level has four subbands: sub-band0 is the low-passed image approximation that is transformed to get next decomposition level; subband1 contains horizontal frequencies, which corresponds to vertical orientation edges in the image; sub-band2 contains both diagonals; and sub-band 3 contains vertical frequencies and corresponds to horizontal orientation edges in the image. Some notation conventions are adopted and introduced later for the global understanding of the paper.

The number of wavelet levels is defined so that the last decomposition level contains details that can be captured by the fovea (1.5-2 degrees of visual angle). This means a frequency range of $0.7-0.5$ pixels per degree, or $0.33-0.25$ cycles per degree. Each level is composed of three oriented sub-bands. The last level additionally contains the approximation image (low-resolution version). Table 1 presents the frequency ranges for each decomposition level.

In the second step of the model, a difference of Gaussian (DoG) modeling the center-surround response of visual cells is applied on each oriented contrast map (wavelet subband). For each location, the center-surround (CS) filter is computed as the difference between current location and the average of surrounding using Eq. 1:
$\operatorname{CS}(x)=|| I(x)\left|-\frac{1}{s} \sum_{k \in S}\right| I(k)||$

where $I(x)$ is the pixel value at location $x, S$ is the surround support, and $s$ the surround surface area. In our implementation, the surround area is a square of $5 \times 5$ pixels for each pyramid level.

Next, the orientation maps from each level are summed together. Because of the multiscale processing, the surface area of a pixel relative to the total image size is greater for low-frequency levels than for high-frequency ones. Therefore, spatial spreading of pixel values is done with an average operation with a circular envelope dependent on the pyramid level. The size of the circular envelope is equal to the size of 1 pixel in the lowest resolution level (1 pixel at level 5 up to 44 pixels at level 0 for the images presented in this study). The aim is to give the same spatial impact on the final map for one pixel regardless of the pyramid level. The level maps (L) are described by Eq. 2. In the remainder of the paper, the analysis levels of wavelet transforms are identified by letter $\mathrm{L}$, from the highest frequency to the lowest one (L0-L5, respectively).

$L(x)=\frac{1}{d} \sum_{k \in D(x)}\left(\sum_{o \in(1,2,3)} \mathrm{CS}_{o}(k)\right)$

$\mathrm{CS}_{o}(k)$ is the center-surround response at location $\mathrm{k}$ for orientation o, $D(x)$ is a disk of radius $1^{\circ}$ centered on $k$, and $d$ its surface area.

The final step is the fusion of these early feature maps. Two fusions are successively applied: levels fusion and color channels fusion. Levels fusion operation is an acrossscale mean using successive bilinear up-sampling and additions $(\oplus)$ of the per-pixel level maps. The channel map (C) is obtained from Eq. 3:

$C(x)=\frac{1}{\mathrm{Nb} \_\mathrm{L}} \oplus_{l} L_{l}(x)$

where $\mathrm{Nb} \_\mathrm{L}$ is the number of decomposition levels used (6 in our case) and $L_{l}$ is the level $l$ map.

YUV components' fusion operation keeps the maximum saliency value between $\mathrm{Y}, \mathrm{U}$, and $\mathrm{V}$ pixels after normalizing with an experimental maximum value, taking into account the difference in amplitude between the three channels (Y: 2500, U\&V: 700) [20, 21]. The output map is finally normalized on an 8-bit integer grayscale image (between 0 and 255).

Table 1 Frequency ranges in wavelet levels

\begin{tabular}{|c|c|c|c|c|c|c|}
\hline Level number & 0 & 1 & 2 & 3 & 4 & 5 \\
\hline Frequency range (cycles per degree) & $10.7-5.3$ & $5.3-2.7$ & $2.7-1.3$ & $1.3-0.7$ & $0.7-0.3$ & $0.3-0.2$ \\
\hline
\end{tabular}


Note that for all the different filters and processing at the border of the image, an infinite extension with mirror is applied. As a result, the model is not centered biased. This consideration is of importance because of the known center bias existing in experimental eye-tracking data [22].

For fair comparison with state-of-the-art models that are center-biased, a number of pixel rows and column from the input picture borders are excluded from the computation. The size of the borders is rescaled in the pyramidal decomposition to avoid a bias in low-resolution levels, i.e., the number of discarded pixels becomes null when a sufficiently high decomposition level is reached.

\section{Experiment Setup}

\section{Stimuli and Category}

This experiment uses four different sets of images belonging to four semantic categories. The stimuli are outdoor color images. These images are grouped into four different categories: Coast, Mountain, Street, and OpenCountry. Each category contains pictures of our environment. Using different categories is motivated by the different conclusions seen in the literature when using different image types [7, 10].

\section{Reference Predefined Pictures}

Stimuli selection process is based on a database proposed by Torralba and Oliva [14]. This database is composed of 10 categories. In order to limit the total number of stimuli in our experiment and still obtain representative categories, only four of them are used here: Coast, Mountain, Street, and OpenCountry. These categories are interesting because their Fourier spectrums are significantly different from each other as illustrated by Fig. 2. This figure shows average spectral profiles and associated distribution histograms. Distribution corresponds to the distance $\mathrm{d}$ from the mean magnitude spectrum normalized by the standard deviation of the category $\left(d(s)=\frac{1}{n \times m} \sum_{n, m} \mid s(n, m)-\right.$ $\mathrm{AS}(n, m) \mid \mathrm{AS}=$ average spectrum). The spectral profiles, which reveal invariant global layouts of the categories, are very distinct from one category to another. Indeed, Coast spectrum presents vertically stretched diamond showing horizontally oriented gist [23], and OpenCountry provides a more proportioned diamond which suggests more equality (on average) in the proportion between horizontal and vertical aspects. Mountain spectrum is isotropic and underlines random aspect of Mountain shapes. Street spectrum is very stretched especially in horizontal axis and in vertical axis, which reveals rectilinear elements characterizing scene with artificial surroundings.

The principle of our selection is to choose our stimuli in order to select images that are close to these Fourier spectrums. It involves a selection process of our own database from Fourier magnitude spectrum distribution of Torralba and Oliva's categories.

\section{Stimuli Pool Constitution}

A first database is composed of 242 color pictures. Each picture has a resolution of $800 \times 600$ pixels. Our categories contain 59 Coast pictures, 44 Street pictures, 61 Mountain, and 78 OpenCountry pictures. Note that these images were selected to present a virgin landscape with very few salient or incongruent objects. The goal is to reduce a bias in gaze allocation due to the presence of locus strongly attracting the gaze such as a bird in OpenCountry and the sky or a cottage in Mountain. The only present objects were constitutive and integrated in surroundings such as a parked car in Street category for example.

Torralba and Oliva's Coast, Street, OpenCountry, and Mountain reference categories contain 360, 292, 410, and
Fig. 2 Representation of the mean spectrum of the 4 used reference Torralba and Oliva's categories with associated distribution histogram. (Picture Database on http://people.csail.mit.edu/ torralba/code/spatialenvelope/)
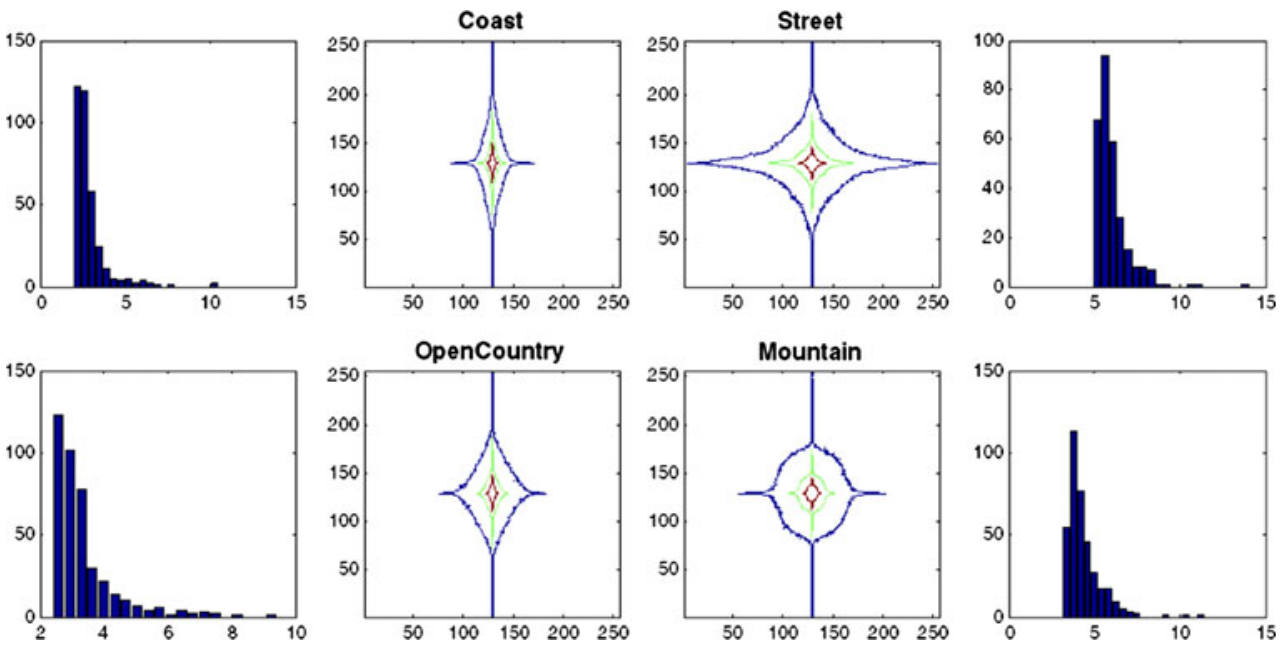
374 images, respectively. Torralba and Oliva's picture database has allowed obtaining reference categorical signal definition (Fourier spectrum central tendencies and mean variability). These categorical attributes are then used to proceed with a stimuli selection across our own database, to obtain a homogeneous and representative database in terms of Fourier spectrum. The selection consists in keeping images having spectrums closer than one standard deviation from the reference category. The distance measure used is the average Euclidean distance $\left(d(s)=\frac{1}{n \times m} \sum_{n, m}|s(n, m)-\mathrm{AS}(n, m)|\right.$ AS = average spectrum). The selection process has rejected $47.5 \%$ on the total of our database of initial stimuli. This selection ensures to have an intracategorical homogeneity and representativeness, based on the selection criteria. The rationale is to use typical features in order to keep general aspects of the categories. The categories contain 30-34 remaining images. Each of the four final categories is constituted of exactly 30 pictures to have equal-sized categories.

\section{Protocol}

Forty voluntary participants ( 22 men and 18 women, mean age $=36.7$ ) of Technicolor Research and Innovation in CessonSévigné (France) participated in this experiment. All subjects had a normal or corrected-to-normal vision. The results from 3 participants have been rejected because recording was incomplete, resulting in a total of 37 observers.

Eye movements were recorded from an infrared reflection capture to detect pupil's location using a SMI RED 50 IView $\mathrm{X}$ system with a $50-\mathrm{Hz}$ sampling.

Stimuli were presented on a screen resolution of $1,280 \times 972$ pixels screen at a distance of $60 \mathrm{~cm}$ $\left(35^{\circ} \times 27^{\circ}\right.$ of visual angle). Each subject recording began with 9 calibration points. All stimuli were presented during $5,500 \mathrm{~ms}$. The presentation order was randomized across participants, and each stimulus was separated by a midgray screen ( $30 \%$ on normalized scale). The subjects were instructed to do natural free viewing of stimuli. Participants were also informed that questions can be asked after the presentation of a stimulus. The questions were only asked in order to keep the subject concentrated on the stimuli. The questions were randomized and were about global content characteristics such as esthetic, chromatic, quality, etc. in order to avoid a subject search strategy.

\section{Data Processing}

The scanpath composed of less than 5 fixations were deleted because they reflect either missing recording or too long fixations associated with visual fatigue. Next, to keep only cognitively valid fixations corresponding to a

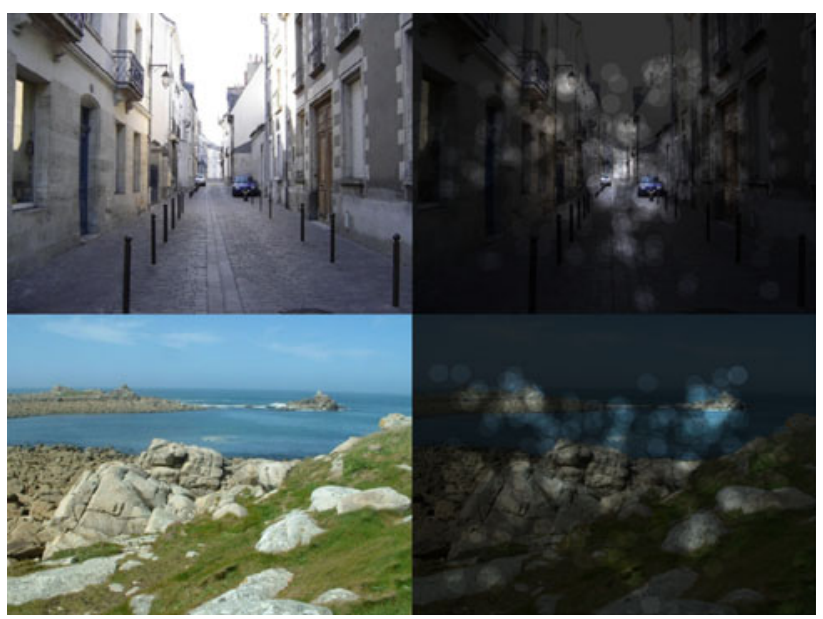

Fig. 3 Example of stimuli for the Street category (top left) and the OpenCountry category (bottom left) with ocular fixation localizations

complete treatment and to reduce measure noise [24], the fixation duration distribution of each scanpath was computed to discard fixations outside the range [average duration $\pm 2 \times$ standard deviation]. This removed $5 \%$ of fixations. After the filtering of fixations, each coordinate was projected with a pixel periphery corresponding to $1.5^{\circ}$ of visual angle (according to the experimental situation) to obtain $4 \times 30$ eye-tracking fixation distribution maps from a set of 37 subjects' ocular fixations. Figure 3 shows two examples of stimuli with superimposed fixations.

\section{Results}

As described in the previous section, an experimental environment has been set up in order to record eye fixations for four scene categories. A simple but efficient computational model of visual attention has also been proposed. In this section, similarity between eye movements collected on the image database and computational saliency maps is investigated. It aims at measuring to what extent one feature at a given resolution may be more or less predictive of attention than another.

This section is composed of two main parts. The proposed model is first benchmarked and compared to existing reference models. Then, attention prediction analysis is performed using only selected features from the model. More specifically, the relative impact of low, medium, and high spatial frequencies on the prediction of attention is examined. One particular interest of this analysis relies on the use of four well-defined categories of outdoor visual scenes.

To conduct this analysis, the degree of similarity between the predicted saliency maps and the visual scanpath is computed. Studies have shown that correlation 

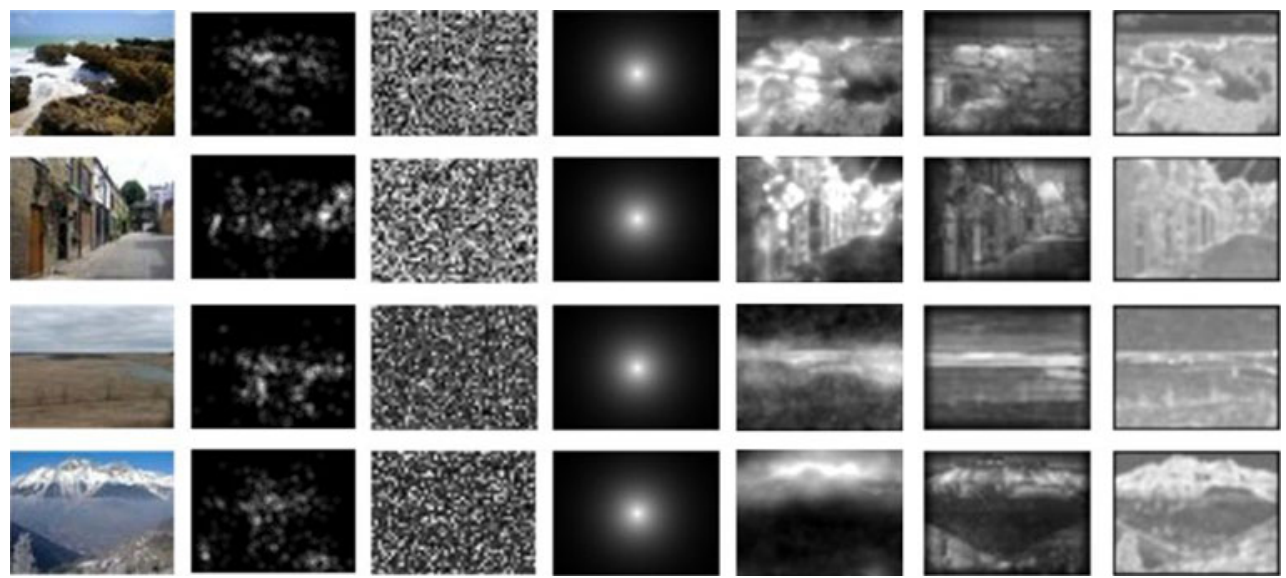

Fig. 4 Saliency maps for all evaluated models. Each row is the results for a particular category. From top to bottom: Coast, Street, OpenCountry, and Mountain. From left to right, original picture,

between fixation locations and signal features decreases in function of the fixation number [4]. In order to measure bottom-up attention only, or at least to limit top-down effects, we use only the first five fixations of visual scanpaths.

Several metrics can be used to assess the degree of similarity between ground truth data and predicted saliency. The normalized scanpath salience metric (NSS) [25] has been chosen for its simplicity and its relevance. It has the advantage to normalize the salience per scanpath: scanpaths with different number of fixations have the same weight. In other words, every observer has the same impact on salience. Moreover, the NSS gives more weight to areas more often fixated.

The NSS is the average value of the saliency map at each fixation normalized per scanpath. First, the saliency map is normalized to have zero mean and unit standard deviation. Then, the per-fixation salience value is computed as the average of the saliency map on the projection of the fixation. A disk with a radius of $1.5^{\circ}$ of visual angle is used to project each fixation. Per-observer NSS is computed as the average of per-fixation salience value along the scanpath. The NSS is the average of the perobserver NSS.

\section{Performance of the Proposed Compared to Existing Models}

The proposed model is compared to four well-known models of the literature. The first two are very simple nonbiologically plausible models: the centered and the random model. The former consists of a centered bidimensional Gaussian. It simply reflects that the center of the screen attracts our gaze whatever the salience. This model is given by the following equation, and an example is depicted in Fig. 4 (fourth column): human fixation distribution map, random map, centered map, proposed saliency map, and Itti's and Bruce's saliency maps

$\mathrm{SM}_{\text {centred }}=e^{-\frac{d\left(s, s_{0}\right)}{\sigma_{c}}}$

where $d\left(s, s_{0}\right)$ is the Euclidean distance between $s$ (the current pixel) and $s_{0}$ (the pixel at the center of the picture), $\sigma_{\mathrm{c}}$ is the standard deviation of the Gaussian and represents the spreading of the saliency distribution, and $\sigma_{\mathrm{c}}$ is set to 100 ( 5 times the number of pixels per degree).

The random model consists of splitting a predicted saliency map into macroblocks of $16 \times 16$ pixels and rearranging them randomly and spatially to recreate a saliency map. An example is provided in Fig. 4 (third column). Note that the random saliency map and the predicted saliency map have exactly the same distribution of salience.

The last two models used in the comparison are the well-known models of Itti and Koch [1] based on the biologically plausible architecture of Koch and Ullman [15], and the one of Bruce and Tsotsos [26] based on ICA learning and information theory. These models are referred to as Itti and Bruce, respectively. Note that these two models are center-biased due to the handling of invalid filter responses at the borders of images, as explained in [27]. As a result, the saliency on the border of the image is erased, as can be seen in Fig. 4. This phenomenon artificially improves the performance of the models because experimental eye-tracking data are known to have a center bias [22]. For a fair comparison, in addition to our proposed model, we used a centered biased version of the same model by discarding an 8-pixel-width border from the input image (proposed + bias). For the sake of comparison, we also present the results associated with the proposed model weighted by the same Gaussian as the centered model (Proposed + Centered).

Figure 5 gives the degree of similarity between different models and human scanpaths in terms of NSS values. Results are consistent with those previously published in 
Fig. 5 Degree of similarity between human scanpath and predicted saliency maps for the whole database. All differences are significant (paired 2-tailed $t$-tests with $P<0.05$ ) unless otherwise stated ( $n s$ no significant difference)

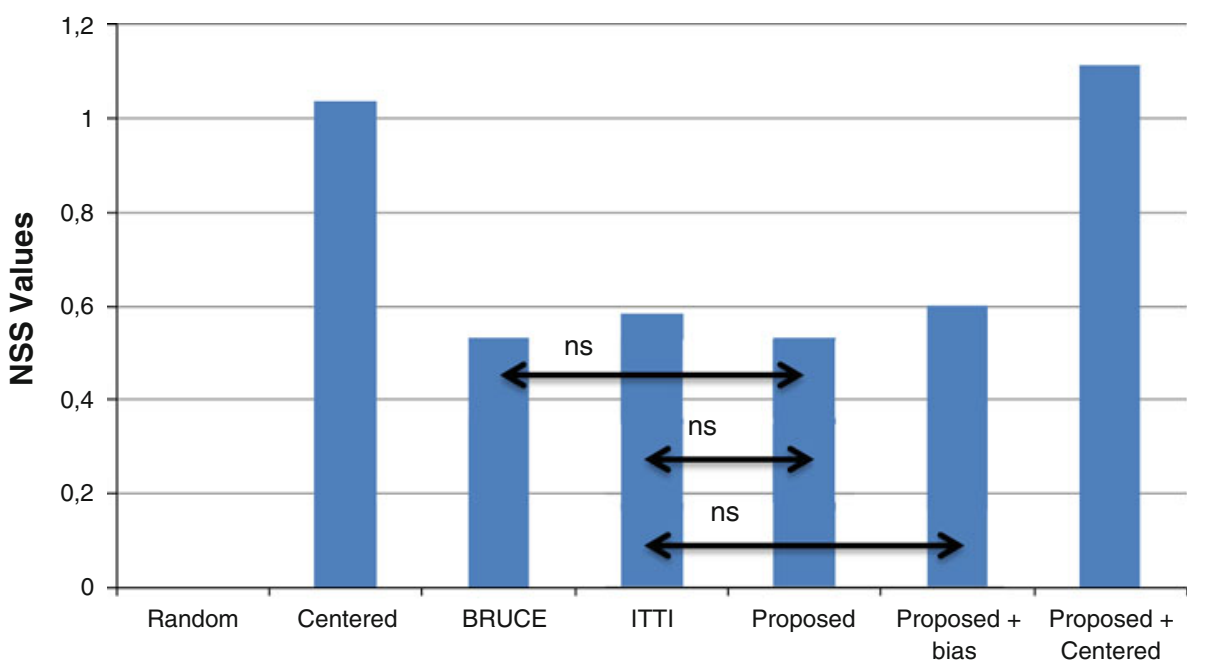

that the centered model gives a better prediction than a signal-based saliency metric [17]. The prediction of the random model is at the chance level (NSS $=0$ ). Results indicate that the proposed model significantly outperforms the model of Bruce and Tsotsos and has higher NSS than Itti and Koch's model when biased with an 8-pixel border. For comparison's interest, the proposed and centered models have been combined. Results are significantly better than the simple centered model.

The proposed model gives higher or comparable performances than state-of-the-art models. This justifies the use of the proposed model for the rest of our experiments. In the remainder of the paper, we use the proposed model with no bias, unless specified. The purpose of this is to allow possible detection of salient area on the border of images. Incidentally, it prevents from using center-biased feature maps.

The goal of the next analysis is to examine whether it is possible to find a better tuning or pooling of the visual features. Is there a preferred spatial frequency? Do the image categories allow discrimination for preferred feature maps?

\section{Predictability According to Frequency Level}

To investigate the relationship between the spatial frequencies of the scenes and the ability to predict the visual fixations, different saliency maps are computed by isolating frequency band factors. For each frequency band, a saliency map is computed. The degree of similarity between these maps and the ground truth is computed with the NSS metric.

The analysis of gaze allocation prediction per frequency range holds by considering the wavelet analysis level without any distinction of color components or orientations. As described in "Methods", it means that the color components and orientations are merged all together.
Firstly, the frequency impact is analyzed without any category distinction. The reader may refer to "Methods" for notations. Figure 6 illustrates the predictive performance (NSS) of our own saliency model according to the frequency band. Results indicate best predictability for mid-frequency band L3 $(0.7-1.3 \mathrm{cpd})$. L3 strongly contributes to the salience prediction. L3 alone is very close to the full model's performances (no significant difference, paired $t$-test $P>0.05$ ). On the other hand, the highest frequency bands alone are significantly more predictive than the random model (NSS $=0$ ), but combined in the full model, they have a relatively weak contribution to salience.

As explained before, we consider different well-defined scene categories to measure potential different behavior according to semantic content. We observe a variation of predictability according to the category. The four categories seem to be not equally predictable (Fig. 7), although only extreme differences are significant.

In Fig. 7, from the comparison with global level, we can observe that two categories are more strongly predictable and two categories are less predictable. Coast is the most predictable category, then OpenCountry, Street at the global level, and finally Mountain for which the results are far below the average.

Figure 8 shows the evolution of the NSS with the frequency band regarding each category. Street and Mountain categories present higher predictability in high and medium frequencies than in low frequencies. The highest NSS for high frequency levels concerns the Street category. Pictures of this category are composed of man-made structures having sharp edges (buildings, windows, etc.) with high contrasts existing across all the frequency bands. Thus, region of interest may be easier to detect than for natural landscapes based on high frequencies only. Previous results showing stronger predictability of high-frequency edge contrast [3,5] seem to be confirmed for man-made scenes. Performance for the Mountain category is below other 
Fig. 6 Gaze prediction according to the spatial frequency for the whole database. This figure illustrates the value of normalized scanpath salience (NSS) versus the frequency bands for the saliency computed from the proposed model. All significant paired $t$-tests $(P<0.05)$ are highlighted with dotted arrows. Note that because there is a dyadic resolution reduction between pyramid levels, the frequency axis is at a $\log$ scale

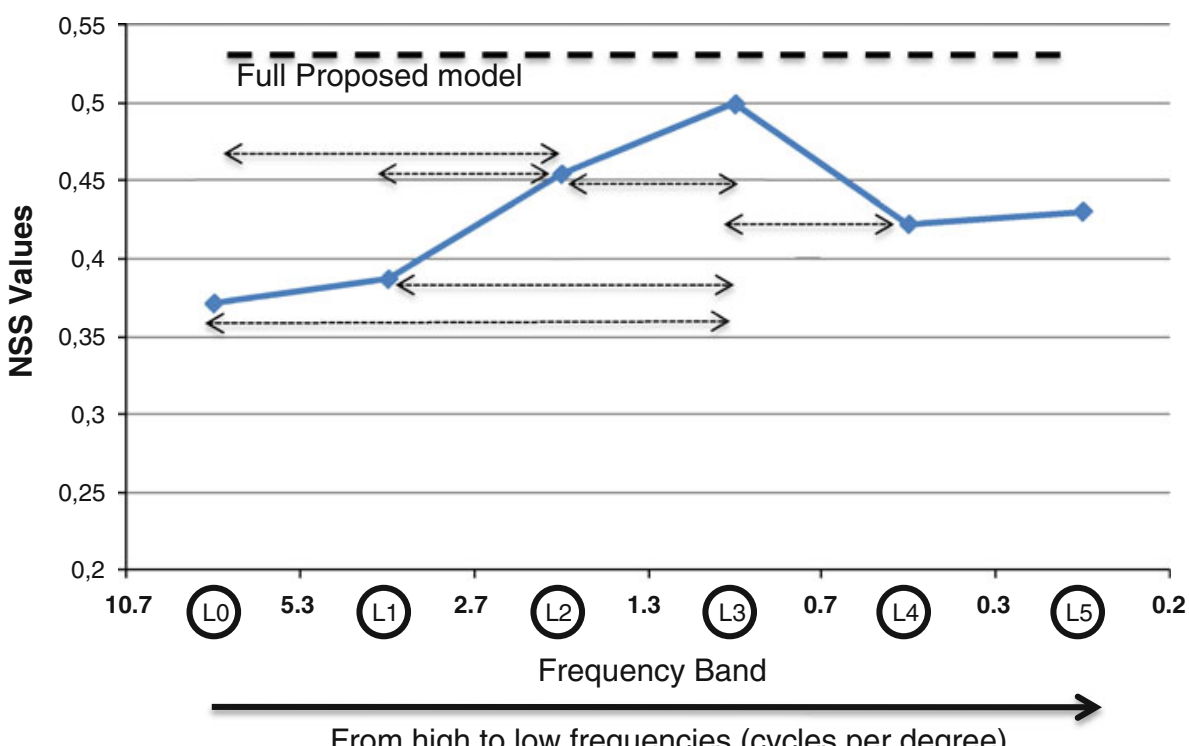

From high to low frequencies (cycles per degree) categories (Fig. 7) and suggests that our model is not able to reliably predict gaze allocation for this type of scene.

The curve obtained from Coast category shows a monotonic increase in its NSS values with decreasing spatial frequency. Low frequencies are thus stronger predictors of gaze allocation for this category.

The curve for OpenCountry clearly shows more predictability in middle frequency (L3). Nevertheless, we note that low-frequency bands (L4 and L5) perform better than high-frequency bands (L0, L1, and L2). Prediction performances for the frequency band L3 are almost the same for three categories and correspond to the average maximum.

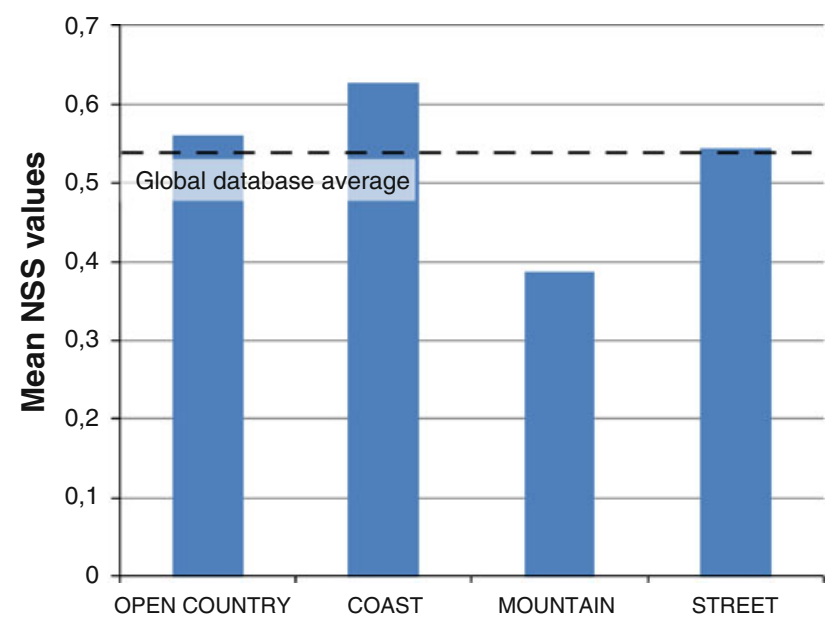

Fig. 7 Gaze prediction according to the scene category (Mean NSS per category). This figure illustrates attention prediction performances according to the category. No difference is significant except Mountain versus Coast (unpaired $t$-tests; $P<0.05$ )
Interestingly, per-frequency band NSS values are on average better for Street than for OpenCountry, whereas using the full model OpenCountry leads to the better performance than Street. This could be due to frequency band pooling that applies the same treatment to all frequency bands. As natural images have a frequency power spectrum biased toward low frequencies, more weight is given to low-frequency features. Furthermore, in the case of Street category, worse performances are obtained when pooling all the frequency bands than when considering each frequency band separately.

\section{Model Improvement Based on Category Differentiation}

Previous results indicate that there is a relationship between salience, scene, and frequency band. Based on this first conclusion, it seems possible to adjust the pooling of the frequency bands in function of the scene type. For each category, we use only the two best frequency levels (L3 and L4 for OpenCountry, L5 and L4 for Coast, L2 and L1 for Mountain, and L2 and L3 for Street) and the other levels are discarded. Incidentally, the suppression of the processing of some levels results in computational simplification, especially at the largest resolution level L0 that gives on average the worst performances and contains the largest amount of data to process. Obtained results are given in Fig. 9.

The modification of the model taking into account category specificities improves the prediction performances globally and for each category. The differences in results are significant only for the global database and Street category. Mountain, Coast, and OpenCountry present a similar slight enhancement, but that was not found to be significant. On the other hand, Street shows a significantly 
Fig. 8 Gaze prediction according to the spatial frequency computed from the proposed model for each category: Coast, OpenCountry, Street, and Mountain. This figure illustrates the value of normalized scanpath salience (NSS) versus the frequency band. The black dotted line corresponds to performances on the whole data set. Significant differences (unpaired $t$-tests) are highlighted with dotted-line arrows

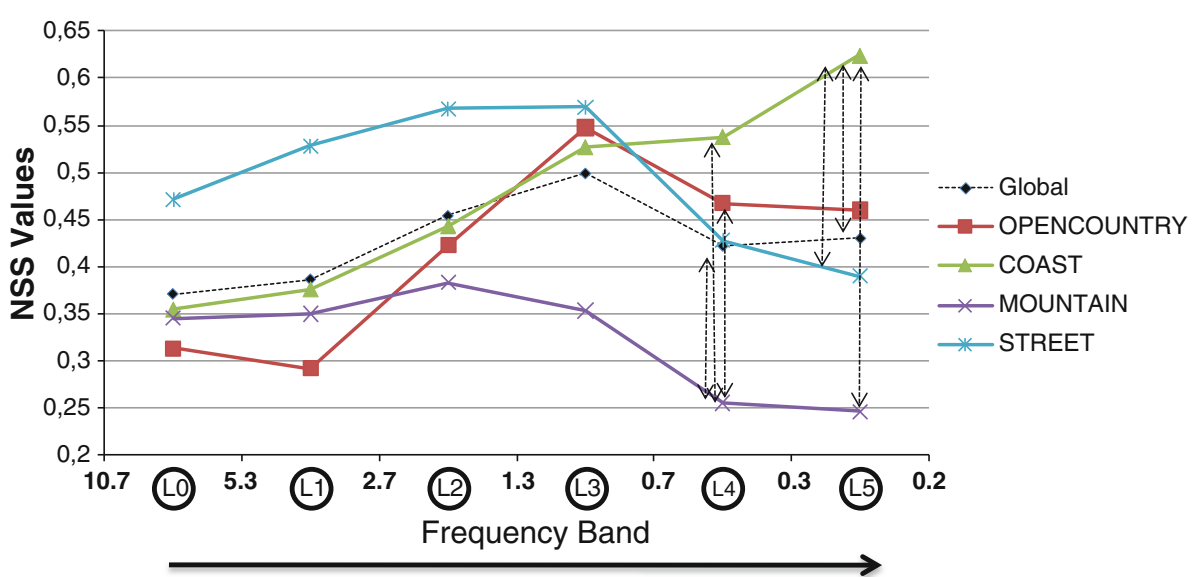

From high to low frequencies (cycles per degree) greater improvement. The fact that the result for the global database is significantly different shows clearly an effect due to the population size. With a larger number of images in each category, these results may be significant for each category. Besides, the significant improvement in the model on the global database has been possible only by combining the improvements in each category.

\section{Discussion}

This study investigated the role of the spatial frequency bands in the determination of visual attention. Fixation points obtained from an eye-tracking experiment have been confronted to the saliency maps computed from the pictures. A visual attention model based on wavelet decomposition has been used to compute salience from each

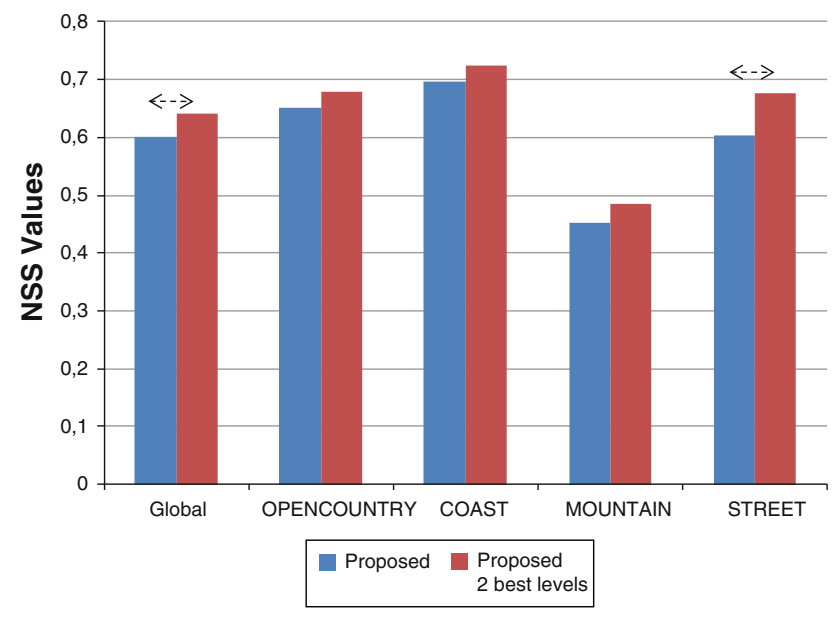

Fig. 9 Degree of similarity between human scanpath and predicted saliency map for the proposed model with 8-pixel border discard and the modified model (with 8-pixel border discard). Significant differences (paired 2-tailed $t$-tests with $P<0.05$ ) are highlighted with dotted arrows wavelet decomposition level. In order to examine the relationship between image content, spatial frequency bands, and gaze allocation prediction, four different picture categories of outdoor scenes have been used. Results indicate that the medium-frequency band-called L3 in our model ( 0.7 to 1.3 cycles per degree (cpd)) — globally contributes the most to gaze attraction. We also found variability among categories. Fixation locations were found to be more predictable using medium to high frequencies (L2: 1.3-2.7 cpd and L3) in man-made Street scenes and low to medium frequencies in natural landscape scenes: Coast: L4 (0.3-0.7 cpd) and L5 (0.3-0.2 cpd); OpenCountry: L3 and L4. Mountain category was slightly more predictable in higher medium frequency (L2).

This result is consistent with previous studies. For instance, Tatler et al. [5] showed that fixated locations, compared to not fixated locations, tended to be more distinctive in the high spatial frequencies. Among others, their stimuli contained indoor and man-made scenes providing particularly sharp edges. While Açik et al. [7] found that luminance contrast explained fixation locations better after low-pass filtering images in the case of natural scenes, and slightly worse in the case of urban scenes.

Several reasons might be proposed in order to explain our results. First, the human visual system has to deal with a large amount of data that the attentional process has to reduce by selecting relevant information. The medium spatial frequencies might be favored by our visual system as a good tradeoff between information collected by the fixation and required attentional resources.

On the one hand, Mannan et al. [28] showed that the fixation durations significantly increased when observers looked at low-pass-filtered visual scenes, compared to unfiltered scenes. It indicates that areas featured only by low spatial frequencies might need more attentional resources than other areas. This is probably due to the subject trying to recognize a blurred object without fine 
details. On the other hand, Fourier power spectrum in natural scenes tends to fall with spatial frequency according to a power law. It means that the scenes are composed mostly of low and medium spatial frequencies, decreasing information quality in high frequency. In order to optimize the tradeoff between attentional resources and quality of perception, it might be a good strategy to start our examination by the medium spatial frequencies. It does not mean that the highest spatial frequencies are not useful. We can make the assumption that in the case where the area under inspection is interesting enough, our visual system can switch toward a more accurate inspection, involving, this time, a fine extraction based on the highest frequencies.

The second point concerns the task. In order to limit topdown factors, observers in this study were instructed to look freely the scene. Therefore, it might be that observers do not pay attention to details, such as the texture of an object. It would probably not be the case if a task was given. For instance, a visual search of a target requires much more attention than a free-view task, and presumably, the detection scanning leads to a strategy involving the processing of finest details provided by high frequencies. In a task-viewing situation, the deployment of visual attention may be more conscious and based on lessredundant features such as high spatial frequencies in accordance with the theory of information [29].

Therefore, we can suppose that medium frequencies are globally more attractive in this study because they constitute an ecological relevance and compromise between attentional resources and information needed when observers are not motivated by challenge of task performing.

Tatler et al. [5] showed that local saliency of fixation loci seems to be constant along the scanpath. They put forward a strategic divergence model: top-down understanding may integrate information delivered by bottom-up driving (saliency) to develop different strategies during scene scanning to influence gaze driving. Saliency comes from an integration of different feature channels and spatial scales. Although saliency keeps its importance over time, different feature channels and spatial scales may be useful according to the different developed strategies along the scanpath [7].

The difference in obtained results between the categories may thus result from an ecological determination of the spatial scale to drive gaze allocation. Indeed, the mediumfrequency band L3 gives the best prediction in average. Street category images are composed of objects such as buildings, cars, and road signs that contain high-frequency edges. The high-frequency driving of gaze allocation may be due to the inspection of these objects. Natural Coast and OpenCountry scenes have been selected to present very few objects.
Even if high frequencies are present in Coast and OpenCountry images, they are more related to landscape texture such as rocks, forests, or grass constituting background than to object edges and do not attract the gaze. Although it represents natural scenes, Mountain category shows more predictability in high frequencies, but the performance values are low. The saliency model may be unable to model factors-or to integrate features-determining gaze driving in this category either because relevant low-level features are missing or because top-down factors may be more determinant for this category.

Finally, the fact that scene categories have distinct frequency spectrums could bias frequency band preponderance, because only available signal can drive fixations. This could explain that the frequency band attracting most the gaze varies in function of image category. However, once integrating category-specific tuning in our saliency model, the performances significantly improved. It means that category-specific spectrums cannot explain alone the role of the spatial frequency bands in the determination of visual attention. This work can thus be used to improve saliency models. A promising way to improve future models would be to combine our approach with other approaches using global semantic and context such as the work of Torralba et al. [30]. The integration of top-down factors, such as local object recognition [31], could also be combined to our approach to improve saliency models.

\section{References}

1. Itti L, Koch C, Niebur E. Model of saliency-based visual attention for rapid scene analysis. IEEE Trans Pattern Anal Mach Intell. 1998;20(11):1254-9.

2. Reinagel P, Zador AM. Natural scene statistics at the centre of gaze. Comput Neural Syst. 1999;10:1-10.

3. Baddeley RJ, Tatler BW. High frequency edges (but not contrast) predict where we fixate: a Bayesian system identification analysis. Vis Res. 2006;46(18):2824-33.

4. Parkhurst D, Law K, Niebur E. Modeling the role of salience in the allocation of overt visual attention. Vis Res. 2002;42:107-23.

5. Tatler BW, Baddeley RJ, Gilchrist ID. Visual correlates of fixation selection: effects of scale and time. Vis Res. 2005;45:643-59.

6. Bruce NDB, Loach DP, Tsotsos JK. Visual correlates of fixation selection: a look at the spatial frequency domain. In: International conference on image processing. 2007.

7. Açik A, Onat S, Schumann F, Einhäuser W, König P. Effects of luminance contrast and its modifications on fixation behavior during free viewing of images from different categories. Vis Res. 2009;49(12):1541-53.

8. Billock VA. Neural acclimation to $1 /$ f spatial frequency spectra in natural images transduced by the human visual system. Phys D Nonlinear Phenom. 2000;137(3-4):379-91.

9. Thorpe S, Fize D, Marlot C. Speed of processing in the human visual system. Nature. 1996;381:520-2.

10. Mermillod M, Guyader N, Chauvin A. The coarse-to-fine hypothesis revisited: evidence from neuro-computational modeling. Brain Cogn. 2005;57(2):151-7. 
11. Séré B, Marendaz C, Hérault J. Nonhomogeneous resolution of images of natural scenes. Perception. 2000;29:1403-12.

12. Schyns P, Oliva A. From blobs to boundary edges: evidence for time- and spatial-scale-dependent scene recognition. Psychol Sci. 1994;5:195-200.

13. Hedgé J. Time course of visual perception: coarse-to-fine perception and beyond. Prog Neurobiol. 2008;84:405-39.

14. Torralba A, Oliva A. Statistics of natural image categories. Comput Neural Syst. 2003;14:391-412.

15. Koch C, Ullman S. Shifts in selective visual attention: towards the underlying neural circuitry. Hum Neurobiol. 1985;4(4): 219-27.

16. Le Meur O, Le Callet P. What we see is most likely to be what matters: visual attention and applications. In: International conference on image processing. 2009. p. 3085-3088.

17. Le Meur O, Le Callet P, Barba D, Thoreau D. A coherent computational approach to model the bottom-up visual attention. IEEE Trans Pattern Anal Mach Intell. 2006;28(5):802-17.

18. Senane H, Saadane A, Barba D. Image coding in the context of a psychovisual image representation with vector quantization. In: ICIP'95. 1995. p. 97-100.

19. Watson $\mathrm{AB}$. The cortex transform: rapid computation of simulated neural images. Comput Vis Graph Image Process. 1987; 39:311-27.

20. Le Meur O, Le Callet P, Barba D. Predicting visual fixations on video based on low-level visual features. Vis Res. 2007;47(19): 2483-98.

21. Ouerhani N, Jost T, Bur A, Hugli H. Cue normalization schemes in saliency-based visual attention models. In: International cognitive vision workshop. 2006.
22. Tatler BW. The central fixation bias in scene viewing: selecting an optimal viewing position independently of motor biases and image feature distributions. J Vis. 2007;7(14):1-17.

23. Oliva A. Gist of the scene. In: Itti L, Rees G, Tsotsos JK, editors. The encyclopedia of neurobiology of attention. Dordrecht: Elsevier; 2005. p. 251-6.

24. Rayner K. Eye movements in reading and information processing: 20 years of research. Psychol Bull. 1998;124(3):372-422.

25. Peters R, Iyer A, Itti L, Koch C. Components of bottom-up gaze allocation in natural images. Vis Res. 2005;45:2397-416.

26. Bruce NDB, Tsotsos JK. Saliency based on information maximization. In: Advances in neural information processing systems. 2006. p. $155-162$.

27. Zhang L, Tong MH, Marks TK, Shan H, Cottrell GW. SUN: a Bayesian framework for saliency using natural statistics. J Vis [Internet]. 2008;8(7). Available from: http://www.journalof vision.org/content/8/7/32.abstract.

28. Mannam SK, Ruddock KH, Wooding DS. Automatic control of saccadic eye movements made in visual inspection of briefly presented 2D images. Spatial Vis. 1995;9:363-86.

29. Bruce NDB. Features that draw visual attention: an information theoretic perspective. Neurocomputing. 2005;65-66:125-33.

30. Torralba A, Oliva A, Castelhano MS, Henderson JM. Contextual guidance of eye movements and attention in real-world scenes: the role of global features in object search. Psychol Rev. 2006;113(4):766-86.

31. Navalpakkam V, Itti L. Modeling the influence of task on attention. Vis Res. 2005;45(2):205-31. 